\title{
Topological solitons in the supersymmetric Skyrme model
}

\author{
Sven Bjarke Gudnason, ${ }^{a}$ Muneto Nitta ${ }^{b}$ and Shin Sasaki ${ }^{c}$ \\ ${ }^{a}$ Institute of Modern Physics, Chinese Academy of Sciences, \\ Lanzhou 730000, China \\ ${ }^{b}$ Department of Physics, and Research and Education Center for Natural Sciences, \\ Keio University, Hiyoshi 4-1-1, Yokohama, Kanagawa 223-8521, Japan \\ ${ }^{c}$ Department of Physics, Kitasato University, \\ Sagamihara 252-0373, Japan \\ E-mail: bjarke@impcas.ac.cn, nitta@phys-h.keio.ac.jp, \\ shin-s@kitasato-u.ac.jp
}

ABSTRACT: A supersymmetric extension of the Skyrme model was obtained recently, which consists of only the Skyrme term in the Nambu-Goldstone (pion) sector complemented by the same number of quasi-Nambu-Goldstone bosons. Scherk-Schwarz dimensional reduction yields a kinetic term in three or lower dimensions and a potential term in two dimensions, preserving supersymmetry. Euclidean solitons (instantons) are constructed in the supersymmetric Skyrme model. In four dimensions, the soliton is an instanton first found by Speight. Scherk-Schwarz dimensional reduction is then performed once to get a 3-dimensional theory in which a 3d Skyrmion-instanton is found and then once more to get a $2 \mathrm{~d}$ theory in which a $2 \mathrm{~d}$ vortex-instanton is obtained. Although the last one is a global vortex it has finite action in contrast to conventional theory. All of them are non-BPS states breaking all supersymmetries.

Keywords: Solitons Monopoles and Instantons, Sigma Models, Supersymmetric Effective Theories

ARXIV EPRINT: 1608.03526 


\section{Contents}

1 Introduction 1

2 The supersymmetric Skyrme model 3

3 Scherk-Schwarz dimensional reductions 5

3.1 Three-dimensional model 5

$\begin{array}{lll}3.2 & \text { Two-dimensional model } & 7\end{array}$

4 Euclidean solitons or instantons $\quad 8$

$4.14 \mathrm{~d}$ pure Skyrme instanton 8

4.2 3d Skyrmion-instanton 9

4.32 d vortex-instanton 11

5 Discussion and conclusion $\quad 13$

$\begin{array}{ll}\text { A BPS property of instanton solutions } & 15\end{array}$

\section{Introduction}

The Skyrme model was originally made as a toy model for baryons where the baryon is made of a soliton in a theory of pions with higher-derivative terms [1,2]. The theory was taken much more serious after Witten showed that the soliton, called the Skyrmion, is exactly the baryon in the large- $N_{c}$ limit of low energy QCD $[3,4]$. Although quite a few phenomenologically appealing results have been achieved in the framework of the Skyrme model, see e.g. [5-9], a withstanding problem is that the binding energies are typically about an order of magnitude too large, compared to experimental data. This motivated a large body of work attempting at lowering the binding energies in Skyrme-like models. One direction is based on a self-dual Yang-Mills theory in five dimensions, dimensionally reduced to four dimensions and in turn giving rise to an infinite tower of vector mesons $[10,11]$. This leads one to search for a theory where the soliton - the Skyrmion — is either a BPS state or saturates a BPS-like energy bound. The original Skyrme model has an energy bound discovered long ago by Faddeev [12]. Sometimes this bound is called a Bogomol'nyi bound [13], which may be misleading because the target space of the Skyrme model is $S^{3}$, which is not Kähler and hence cannot be supersymmetrized. The mentioned energy bound is, however, not saturable unless the space is isometric to a 3-sphere [14]. A different model was constructed later, which is by now called the BPS Skyrme model as it has a saturable energy bound $[15,16]$. Supersymmetrizing the BPS Skyrme model was attempted in [17], which however is not possible due to the fact that its target space is not Kähler [18]. 
Supersymmetrizing the Skyrme model was attempted early on, in the literature [19]. Several problems were encountered along the way. The first problem of $S^{3}$ not being Kähler was remedied by switching the target space to $\mathbb{C} P^{1}$, which however is the target space of a baby Skyrme model rather than of the Skyrme model. The next problem is that unless care is taken in constructing the model, one will encounter the auxiliary field problem; i.e. the auxiliary field, $F$, in the chiral multiplet will have derivatives acting on it and in turn making it a dynamical field. ${ }^{1}$ Finally, even circumventing the auxiliary field problem, the supersymmetrized Skyrme-like term turned out to have four time derivatives [19, 21], making a Hamiltonian formulation impossible. The later attempt at supersymmetrizing the baby-Skyrme model also arrived at the same type of Lagrangian with four time derivatives [21].

Thirty years after the problem was laid out in the seminal paper [19] not much progress on constructing the Skyrme term was made, until a systematic investigation of the supersymmetric four-derivative term without the auxiliary field problem [22-25] lead to the idea that a non-Abelian nontrivial solution to the non-dynamic auxiliary field equation could produce a supersymmetric Skyrme term. This was carried out in [26] and indeed the nontrivial solution to the auxiliary field equation gave a term whose Nambu-Goldstone (NG) submanifold is exactly the Skyrme term. The construction is based on complexifying $\mathrm{SU}(2)$ to $\mathrm{SL}(2, \mathbb{C})$, which in turn gives rise to three new bosonic degrees of freedom called quasi-NG bosons [27]; this complexification is inevitable due to nonlinear realization theory. Once the quasi-NG bosons are turned on, the supersymmetric Skyrme term is more complicated and does possess four time derivatives, again not allowing for a Hamiltonian formulation. The restriction to the NG submanifold, however, eliminates the four time derivatives and as mentioned above, yields exactly the Skyrme term. A twist compared to the non-supersymmetric Skyrme model, is that if one tries to turn on a standard kinetic (Dirichlet) term, the nontrivial solution to the auxiliary field equation will simply eliminate it, leaving just a potential term for the quasi-NG bosons. Introducing a superpotential has not been carried out yet.

It requires an attractive term in the Lagrangian in order for a Skyrmion to be stabilized - i.e. a nontrivial solution to the virial equation due to Derrick's theorem. In the conventional Skyrme model, the kinetic term and the Skyrme term is balanced. In this paper we will induce a kinetic term in the theory by performing Scherk-Schwarz (SS) [28, 29] dimensional reduction (DR) in order to construct a Skyrmion, although our Skyrmion is rather an instanton in three Euclidean dimensions. Although the original formulation breaks supersymmetry due to twisting of the $R$-symmetry, supersymmetry preserving dimensional reductions are possible, see e.g. [30].

We derive 3-dimensional and 2-dimensional Lagrangians by supersymmetry preserving SS dimensional reductions and in turn construct solitons in all dimensions from two through four. The first soliton is the instanton found by Speight [31] in the so-called pure Skyrme model - which is exactly the NG part of the supersymmetric Skyrme model. Next, we construct a 3-dimensional Skyrmion-instanton in the once SS reduced theory. Finally, we

\footnotetext{
${ }^{1}$ For a recent work in this direction, however, see [20].
} 
create a vortex-instanton in the twice SS anisotropically reduced Euclidean two-dimensional theory. All of these instantons are non-BPS states, breaking all supersymmetries.

The plan of the paper is as follows. We start by reviewing the supersymmetric Skyrme model in section 2. In section 3 we construct the SS dimensionally reduced Lagrangians that we will use to construct the lower-dimensional solitons in section 4. Finally, we conclude with a discussion in section 5. The appendix discusses the BPS property of the solitons.

\section{The supersymmetric Skyrme model}

We will begin by reviewing the supersymmetric Skyrme model, found in [26]. The construction of fourth-order derivative terms in supersymmetry - without the auxiliary field problem - is based on the Lagrangian

$$
\mathcal{L}=\int d^{4} \theta K\left(\Phi, \Phi^{\dagger}\right)+\frac{1}{16} \int d^{4} \theta \Lambda_{A B \bar{C} \bar{D}}\left(\Phi, \Phi^{\dagger}\right) D^{\alpha} \Phi^{A} D_{\alpha} \Phi^{B} \bar{D}_{\dot{\alpha}} \Phi^{\bar{C} \dagger} \bar{D}^{\dot{\alpha}} \Phi^{\bar{D} \dagger}
$$

where $K\left(\Phi, \Phi^{\dagger}\right)$ is a Kähler potential and $\Lambda_{A B \bar{C} \bar{D}}\left(\Phi, \Phi^{\dagger}\right)$ is a Kähler tensor with the indices $A, B$ and $\bar{C}, \bar{D}$ symmetrized pairwise. The chiral superfields $\Phi^{A}$ are then combined with a nonlinear sigma model field

$$
M=\exp \left(i \Phi^{A} t^{A}\right) \in G^{\mathbb{C}} / \hat{H}
$$

taking value in the coset relevant for chiral symmetry breaking:

$$
G^{\mathbb{C}} / \hat{H} \simeq \mathrm{SU}(N)^{\mathbb{C}}=G^{\mathbb{C}} / H^{\mathbb{C}} \simeq \mathrm{SL}(N, \mathbb{C}) \simeq T^{*} \mathrm{SU}(N) .
$$

Here, $\hat{H}$ is the complex isotropy group and not necessarily equal to $H^{\mathbb{C}}$ but can be larger in general [32-34].

The superfields $\Phi^{A}$ are composed of NG bosons $\pi^{A}$, quasi-NG bosons $\sigma^{A}$, quasi-NG fermions $\psi^{A}$ and auxiliary fields $F^{A}$ as

$$
\Phi^{A}(y, \theta)=\pi^{A}(y)+i \sigma^{A}(y)+\theta \psi^{A}(y)+\theta^{2} F^{A}(y) .
$$

Our case of chiral symmetry breaking falls into the class of maximally realized supersymmetrizations (and therefore $\hat{H}=H^{\mathbb{C}}$ ) which means that the number of quasi-NG bosons is equal to the number of NG bosons [27, 35]. The Kähler potential, $K\left(\Phi, \Phi^{\dagger}\right)$, used for constructing the supersymmetric Skyrme model [26] is

$$
K=f_{\pi}^{2} \operatorname{Tr} M M^{\dagger},
$$

and the $(2,2)$ Kähler tensor, $\Lambda_{A B \bar{C} \bar{D}}\left(\Phi, \Phi^{\dagger}\right)$, is implicitly defined by

$$
\begin{aligned}
& \int d^{4} \theta \Lambda_{A B \bar{C} \bar{D}}\left(\Phi, \Phi^{\dagger}\right) D^{\alpha} \Phi^{A} D_{\alpha} \Phi^{B} \bar{D}_{\dot{\alpha}} \Phi^{\bar{C} \dagger} \bar{D}^{\dot{\alpha}} \Phi^{\bar{D} \dagger} \\
& =\int d^{4} \theta \Lambda\left(M, M^{\dagger}\right) \operatorname{Tr}\left[D^{\alpha} M \bar{D}_{\dot{\alpha}} M^{\dagger} D_{\alpha} M \bar{D}^{\dot{\alpha}} M^{\dagger}\right] .
\end{aligned}
$$


Then the bosonic part of the Lagrangian is

$$
\begin{aligned}
\mathcal{L}_{b}^{(4)}= & f_{\pi}^{2} \operatorname{Tr}\left[-M_{\mu} M^{\mu}+F^{\dagger} F\right] \\
& +\Lambda\left(M, M^{\dagger}\right) \operatorname{Tr}\left[M_{\mu}^{\dagger} M_{\nu} M^{\mu \dagger} M^{\nu}+\left(F^{\dagger} F\right)^{2}-M_{\mu}^{\dagger} M^{\mu} F^{\dagger} F-M_{\mu}^{\dagger} M^{\mu} F F^{\dagger}\right],
\end{aligned}
$$

where we have introduced the short notation $M_{\mu} \equiv \partial_{\mu} M$ and $f_{\pi}$ is the pion decay constant. The first term is the ordinary kinetic (Dirichlet) term with two time derivatives while the second term is a higher derivative correction. The Kähler tensor $\Lambda_{A B \bar{C} \bar{D}}$ in (2.1) is determined by the $G$-invariant function $\Lambda\left(M, M^{\dagger}\right)$ through the relation (2.2). The equations of motion for the auxiliary fields are

$$
\begin{aligned}
f_{\pi}^{2} \Lambda^{-1} F+2 F F^{\dagger} F-F M_{\mu}^{\dagger} M^{\mu}-M_{\mu} M^{\dagger \mu} F & =0, \\
f_{\pi}^{2} \Lambda^{-1} F^{\dagger}+2 F^{\dagger} F F^{\dagger}-M_{\mu}^{\dagger} M^{\mu} F^{\dagger}-F^{\dagger} M_{\mu} M^{\dagger \mu} & =0 .
\end{aligned}
$$

The Lagrangian (2.7) avoids the auxiliary field problem and hence the auxiliary field equation is algebraic; it is, nevertheless, a nontrivial matrix equation.

Two consistent possibilities of solutions to the equations (2.8) arise if we do not introduce a superpotential. The first corresponds to the trivial solution $F=0$ which is called the canonical branch. The on-shell Lagrangian on the canonical branch is obtained straightforwardly from eq. (2.7):

$$
\mathcal{L}_{b}^{(4)}=-f_{\pi}^{2} \operatorname{Tr} M_{\mu}^{\dagger} M^{\mu}+\Lambda\left(M, M^{\dagger}\right) \operatorname{Tr}\left[M_{\mu}^{\dagger} M_{\nu} M^{\mu \dagger} M^{\nu}\right] .
$$

However, this term does not reduce to the Skyrme term when the quasi-NG fields are set to zero and it also contains four time-derivatives.

The second is the non-canonical branch associated with the non-trivial solutions $F \neq 0$. The non-canonical branch for the theory (2.7) without a superpotential was found explicitly in [26] for the $\mathrm{SU}(2)$ case

$$
\begin{aligned}
\mathcal{L}_{b}^{(4)}= & \frac{\Lambda\left(M, M^{\dagger}\right)}{2}\left\{\operatorname{Tr}\left[2 M_{\mu}^{\dagger} M_{\nu} M^{\mu \dagger} M^{\nu}-\frac{1}{2} M_{\mu}^{\dagger} M^{\mu} M_{\nu}^{\dagger} M^{\nu}-\frac{1}{2} M_{\mu} M^{\mu \dagger} M_{\nu} M^{\nu \dagger}\right] \quad\right. \\
& -\frac{1}{2}\left(\operatorname{Tr}\left[M_{\mu} M^{\mu \dagger}\right]\right)^{2}-\operatorname{Tr}\left[\frac{f_{\pi}^{4}}{2 \Lambda^{2}\left(M, M^{\dagger}\right)} \mathbf{1}_{2}\right] \\
& \mp \sqrt{\left(\operatorname{Tr}\left[M_{\mu}^{\dagger} M^{\mu} M_{\nu}^{\dagger} M^{\nu}\right]-\frac{1}{2}\left(\operatorname{Tr}\left[M_{\mu} M^{\mu \dagger}\right]\right)^{2}\right)\left(\operatorname{Tr}\left[M_{\mu} M^{\mu \dagger} M_{\nu} M^{\nu \dagger}\right]-\frac{1}{2}\left(\operatorname{Tr}\left[M_{\mu} M^{\mu \dagger}\right]\right)^{2}\right)} .
\end{aligned}
$$

We note that the ordinary second-order kinetic term is canceled on the non-canonical branch. Although the term with four time derivatives does not cancel in general when the quasi-NG fields are turned on, the above Lagrangian simplifies exactly to the Skyrme term when they are turned off

$$
\left.\mathcal{L}_{b}^{(4)}\right|_{M=U}=\Lambda \operatorname{Tr}\left[U_{\mu}^{\dagger} U_{\nu} U^{\mu \dagger} U^{\nu}-U_{\mu}^{\dagger} U^{\mu} U_{\nu}^{\dagger} U^{\nu}\right]-\operatorname{Tr}\left[\frac{f_{\pi}^{4}}{4 \Lambda} \mathbf{1}_{2}\right] .
$$

The prefactor $\Lambda\left(M, M^{\dagger}\right)$ is a function of $G$-invariants and thus when restricting to the NG submanifold, $\Lambda\left(U U^{\dagger}=\mathbf{1}_{2}\right)=\Lambda$ becomes a constant. The effect of adding the kinetic 
(Dirichlet) term thus only had the effect of inducing the potential (the last term in the above Lagrangian). If we consider a $G$-invariant theory, $\Lambda$ must be a constant and thus the potential is just a c-number that we can ignore.

The upshot is thus that the Kähler potential cannot induce a kinetic term or a potential if $G$-invariance is preserved. In the next section, we will perform Scherk-Schwarz dimensional reductions in order to induce a kinetic term.

\section{Scherk-Schwarz dimensional reductions}

Our starting point will be the supersymmetric Skyrme model (2.10) in 4-dimensional Euclidean space. We will keep the $G$-invariance intact throughout this paper, so the addition of the kinetic term will only induce a constant and hence not affect the equations of motion. Therefore we will simply work with only the fourth-order derivative term and ignore the latter constant by setting $f_{\pi}=0$. For simplicity, we will restrict to the NG submanifold before performing Scherk-Schwarz (SS) dimensional reduction (DR) and it will prove convenient to change notation to a 4 -vector $\mathbf{n}=\left\{n^{1}, n^{2}, n^{3}, n^{4}\right\}$ as

$$
U=\mathbf{1}_{2} n^{4}+i n^{a} \tau^{a}
$$

where $\tau^{a}$ are the Pauli matrices and the fields satisfy $\mathbf{n} \cdot \mathbf{n}=1$. Using this notation, the NG restricted Skyrme model on Euclidean four-space, $\mathbb{R}^{4}$, reads

$$
\mathcal{L}_{4 d, b}^{(4)}=\frac{1}{4}\left(\mathbf{n}_{\mu} \cdot \mathbf{n}_{\mu}\right)^{2}-\frac{1}{4}\left(\mathbf{n}_{\mu} \cdot \mathbf{n}_{\nu}\right)^{2},
$$

where we have defined $\mathbf{n}_{\mu}=\partial_{\mu} \mathbf{n}$ and lowered all the indices since the Euclidean metric is just the identity matrix. We have also set $\Lambda=1 / 16$ for convenience. The Lagrangian (3.2) admits a symmetry under the transformation $\mathbf{n}^{\prime}=O \mathbf{n}, O \in \mathrm{SO}(4)$ which will be utilized for the SS reduction.

\section{$3.1 \quad$ Three-dimensional model}

We are now ready to perform the first SSDR by compactifying the fourth coordinate. We will use the coordinates $x^{\mu}$ with $\mu=1,2,3,4$ where we have Wick-rotated the time coordinate, and SS dimensional reduction along $x^{4} \sim x^{4}+2 \pi R_{4}$ is carried out as follows

$$
\mathbf{n}\left(x^{\mu}\right)=O\left(x^{4}\right) \mathbf{N}\left(x^{a}\right),
$$

where $a=1,2,3$ runs over the non-compactified dimensions and the matrix

$$
O\left(x^{4}\right)=-O\left(x^{4}+2 \pi R_{4}\right) \equiv\left(\begin{array}{cccc}
\cos \frac{\mathrm{m}_{4,1} x^{4}}{2 R_{4}} & -\sin \frac{\mathrm{m}_{4,1} x^{4}}{2 R_{4}} & 0 & 0 \\
\sin \frac{\mathrm{m}_{4,1} x^{4}}{2 R_{4}} & \cos \frac{\mathrm{m}_{4,1} x^{4}}{2 R_{4}} & 0 & 0 \\
0 & 0 & \cos \frac{\mathrm{m}_{4,2} x^{4}}{2 R_{4}} & -\sin \frac{\mathrm{m}_{4,2} x^{4}}{2 R_{4}} \\
0 & 0 & \sin \frac{\mathrm{m}_{4,2} x^{4}}{2 R_{4}} & \cos \frac{\mathrm{m}_{4,2} x^{4}}{2 R_{4}}
\end{array}\right),
$$


where $\mathrm{m}_{4,1} \in \mathbb{Z}_{\neq 0}$ and $\mathrm{m}_{4,2} \in \mathbb{Z}_{\neq 0}$ are two nonzero Kaluza-Klein (KK) integers describing towers of higher-momentum states along the compactified circle. Notice that we have applied twisted boundary conditions (TBC) such that

$$
\mathbf{n}\left(x^{4}+2 \pi R_{4}\right)=\epsilon\left(\mathrm{m}_{4,1}, \mathrm{~m}_{4,2}\right) \mathbf{n}\left(x^{4}\right),
$$

with

$$
\epsilon\left(\mathrm{m}_{4,1}, \mathrm{~m}_{4,2}\right)=\left(\begin{array}{llll}
(-1)^{\mathrm{m}_{4,1}} & & & \\
& (-1)^{\mathrm{m}_{4,1}} & & \\
& & (-1)^{\mathrm{m}_{4,2}} & \\
& & & (-1)^{\mathrm{m}_{4,2}}
\end{array}\right),
$$

which is just an $\mathrm{SO}(4)$ transformation of the fields.

After the dust settles, we obtain

$$
\begin{aligned}
\mathcal{L}_{3 d, b}^{(4)}= & \frac{\pi R_{4}}{2}\left[\left(\mathbf{N}_{a} \cdot \mathbf{N}_{a}\right)^{2}-\left(\mathbf{N}_{a} \cdot \mathbf{N}_{b}\right)^{2}\right] \\
& +\frac{\pi}{4 R_{4}}\left[\mathrm{~m}_{4,1}^{2}+\left(\mathrm{m}_{4,2}^{2}-\mathrm{m}_{4,1}^{2}\right)\left\{\left(N^{3}\right)^{2}+\left(N^{4}\right)^{2}\right\}\right] \mathbf{N}_{a} \cdot \mathbf{N}_{a} \\
& -\frac{\pi}{4 R_{4}}\left[\mathrm{~m}_{4,1}\left(N^{1} N_{a}^{2}-N^{2} N_{a}^{1}\right)+\mathrm{m}_{4,2}\left(N^{3} N_{a}^{4}-N^{4} N_{a}^{3}\right)\right]^{2},
\end{aligned}
$$

where we again have defined the notation $\mathbf{N}_{a}=\partial_{a} \mathbf{N}$ and used the relation $\mathbf{N} \cdot \mathbf{N}=1$. If we set the two integers $\mathrm{m}_{4,1}$ and $\mathrm{m}_{4,2}$ equal to each other and to $\mathrm{m}_{4} \in \mathbb{Z}_{\neq 0}$, the SS dimensionally reduced Lagrangian simplifies to

$$
\begin{aligned}
\mathcal{L}_{3 d, b}^{(4)}= & \frac{\pi R_{4}}{2}\left[\left(\mathbf{N}_{a} \cdot \mathbf{N}_{a}\right)^{2}-\left(\mathbf{N}_{a} \cdot \mathbf{N}_{b}\right)^{2}\right]+\frac{\mathrm{m}_{4}^{2} \pi}{4 R_{4}} \mathbf{N}_{a} \cdot \mathbf{N}_{a} \\
& -\frac{\mathrm{m}_{4}^{2} \pi}{4 R_{4}}\left[N^{1} N_{a}^{2}-N^{2} N_{a}^{1}+N^{3} N_{a}^{4}-N^{4} N_{a}^{3}\right]^{2}
\end{aligned}
$$

Then the last term in the first line is a kinetic term with a prefactor of the KK mass in the 3-dimensional Euclidean theory. We stress that the SS reduction of the fourth-order derivative term of the BPS Skyrme model produces the usual (second-order derivatives term) kinetic term. This is in contradistinction to the ordinary case where the potential term appears by SS reduction from the usual kinetic term.

Let us note that the lowest energy state comes from the lowest KK mode and thus the compactified momenta correspond to the integers $\mathrm{m}_{4,1}= \pm 1$ and $\mathrm{m}_{4,2}= \pm 1$. It is clear from the SS reduced Lagrangian (3.8) that the overall sign of the two integers is physically unobservable. One may naively think that the relative sign could matter, but renaming the two fields $\left\{N^{3}, N^{4}\right\} \rightarrow\left\{N^{4}, N^{3}\right\}$ compensates a relative minus sign.

A further remark about the KK momenta is in store. Because $\pi_{1}(\mathrm{SU}(2))$ is trivial, higher even momentum numbers $\mathrm{m}_{4}$ may be metastable or unstable and could decay to $\mathrm{m}_{4}=0$ while for odd integers, they may decay to the states with $\mathrm{m}_{4}= \pm 1$. This holds for both the KK integers. The states with minimum energy are thus $\mathrm{m}_{4}=-1,0,1$, where the $\mathrm{m}_{4}=0$ is distinguished from $\mathrm{m}_{4}= \pm 1$ by the boundary conditions. We will focus on the latter in this paper. 


\subsection{Two-dimensional model}

Now we will perform another consecutive SS dimensional reduction, but along $x^{3} \sim x^{3}+$ $2 \pi R_{3}$ as

$$
\mathbf{N}\left(x^{a}\right)=\widetilde{O}\left(x^{3}\right) \mathbf{M}\left(x^{i}\right),
$$

where $i=1,2$ runs over the non-compactified dimensions and

$$
\widetilde{O}\left(x^{3}\right)=-\widetilde{O}\left(x^{3}+2 \pi R_{3}\right) \equiv\left(\begin{array}{cccc}
\cos \frac{\mathrm{m}_{3,1} x^{3}}{2 R_{3}} & -\sin \frac{\mathrm{m}_{3,1} x^{3}}{2 R_{3}} & 0 & 0 \\
\sin \frac{\mathrm{m}_{3,1} x^{3}}{2 R_{3}} & \cos \frac{\mathrm{m}_{3,1} x^{3}}{2 R_{3}} & 0 & 0 \\
0 & 0 & \cos \frac{\mathrm{m}_{3,2} x^{3}}{2 R_{3}} & -\sin \frac{\mathrm{m}_{3,2} x^{3}}{2 R_{3}} \\
0 & 0 & \sin \frac{\mathrm{m}_{3,2} x^{3}}{2 R_{3}} & \cos \frac{\mathrm{m}_{3,2} x^{3}}{2 R_{3}}
\end{array}\right),
$$

where $\mathrm{m}_{3,1} \in \mathbb{Z}_{\neq 0}$ is a nonzero integer while $\mathrm{m}_{3,2} \in \mathbb{Z}$ is an integer; we allow it to be vanishing in order to get an anisotropic SS dimensional reduction (this does not correspond to a compactified momentum not being quantized, but merely formally to the option of making the last two fields independent of the circle coordinate). The TBC are then $\mathbf{N}\left(x^{3}+\right.$ $\left.2 \pi R_{3}\right)=\epsilon\left(\mathrm{m}_{3,1}, \mathrm{~m}_{4,2}\right) \mathbf{N}\left(x^{3}\right)$, with $\epsilon$ again given by eq. (3.6).

Starting now from the 3-dimensional Euclidean Lagrangian (3.7), we get

$$
\begin{aligned}
\mathcal{L}_{2 d, b}^{(4)}= & \pi^{2} R_{3} R_{4}\left[\left(\mathbf{M}_{i} \cdot \mathbf{M}_{i}\right)^{2}-\left(\mathbf{M}_{i} \cdot \mathbf{M}_{j}\right)^{2}\right] \\
+ & \frac{\pi^{2}}{2}\left[\mathrm{~m}_{4,1}^{2} \frac{R_{3}}{R_{4}}+\mathrm{m}_{3,1}^{2} \frac{R_{4}}{R_{3}}\right. \\
& \left.\quad+\left\{\left(\mathrm{m}_{4,2}^{2}-\mathrm{m}_{4,1}^{2}\right) \frac{R_{3}}{R_{4}}+\left(\mathrm{m}_{3,2}^{2}-\mathrm{m}_{3,1}^{2}\right) \frac{R_{4}}{R_{3}}\right\}\left[\left(M^{3}\right)^{2}+\left(M^{4}\right)^{2}\right]\right] \mathbf{M}_{i} \cdot \mathbf{M}_{i} \\
& -\frac{\pi^{2}}{2}\left(\mathrm{~m}_{4,1}^{2} \frac{R_{3}}{R_{4}}+\mathrm{m}_{3,1}^{2} \frac{R_{4}}{R_{3}}\right)\left(M^{1} M_{i}^{2}-M^{2} M_{i}^{1}\right)^{2} \\
- & \frac{\pi^{2}}{2}\left(\mathrm{~m}_{4,2}^{2} \frac{R_{3}}{R_{4}}+\mathrm{m}_{3,2}^{2} \frac{R_{4}}{R_{3}}\right)\left(M^{3} M_{i}^{4}-M^{4} M_{i}^{3}\right)^{2} \\
- & \pi^{2}\left(\mathrm{~m}_{4,1} \mathrm{~m}_{4,2} \frac{R_{3}}{R_{4}}+\mathrm{m}_{3,1} \mathrm{~m}_{3,2} \frac{R_{4}}{R_{3}}\right)\left(M^{1} M_{i}^{2}-M^{2} M_{i}^{1}\right)\left(M^{3} M_{i}^{4}-M^{4} M_{i}^{3}\right) \\
+ & \frac{\pi^{2}}{2 R_{3} R_{4}}\left(\mathrm{~m}_{4,1}^{2} \mathrm{~m}_{3,2}^{2}+\mathrm{m}_{4,2}^{2} \mathrm{~m}_{3,1}^{2}-2 \mathrm{~m}_{3,1} \mathrm{~m}_{3,2} \mathrm{~m}_{4,1} \mathrm{~m}_{4,2}\right) \\
\quad & \quad\left[\left(M^{1}\right)^{2}+\left(M^{2}\right)^{2}\right]\left[\left(M^{3}\right)^{2}+\left(M^{4}\right)^{2}\right],
\end{aligned}
$$

where $\mathbf{M}_{i}=\partial_{i} \mathbf{M}$. In the case the two momenta on each compactified circle are equal, viz. when $\mathrm{m}_{3,1}=\mathrm{m}_{3,2}=\mathrm{m}_{3}$ and $\mathrm{m}_{4,1}=\mathrm{m}_{4,2}=\mathrm{m}_{4}$, a great simplification occurs

$$
\begin{aligned}
\mathcal{L}_{2 d, b}^{(4)}= & \pi^{2} R_{3} R_{4}\left[\left(\mathbf{M}_{i} \cdot \mathbf{M}_{i}\right)^{2}-\left(\mathbf{M}_{i} \cdot \mathbf{M}_{j}\right)^{2}\right]+\frac{\pi^{2}}{2}\left[\mathrm{~m}_{4}^{2} \frac{R_{3}}{R_{4}}+\mathrm{m}_{3}^{2} \frac{R_{4}}{R_{3}}\right] \mathbf{M}_{i} \cdot \mathbf{M}_{i} \\
& -\frac{\pi^{2}}{2}\left(\mathrm{~m}_{4}^{2} \frac{R_{3}}{R_{4}}+\mathrm{m}_{3}^{2} \frac{R_{4}}{R_{3}}\right)\left(M^{1} M_{i}^{2}-M^{2} M_{i}^{1}+M^{3} M_{i}^{4}-M^{4} M_{i}^{3}\right)^{2} .
\end{aligned}
$$


However, we can see that this simplification also eliminates the potential, i.e. only derivative terms remain.

In order to get a relatively simple Lagrangian with a potential, let us consider the case where the first compactification has equal momenta $\left(\mathrm{m}_{4,1}=\mathrm{m}_{4,2}=\mathrm{m}_{4}\right)$ while the second compactification has one nonvanishing momentum $\mathrm{m}_{3,1}=\mathrm{m}_{3} \neq 0$ and one vanishing $\mathrm{m}_{3,2}=0$. This will simplify the Lagrangian (3.11) to

$$
\begin{aligned}
\mathcal{L}_{2 d, b}^{(4)}= & \pi^{2} R_{3} R_{4}\left[\left(\mathbf{M}_{i} \cdot \mathbf{M}_{i}\right)^{2}-\left(\mathbf{M}_{i} \cdot \mathbf{M}_{j}\right)^{2}\right] \\
& +\frac{\pi^{2}}{2}\left[\mathrm{~m}_{4}^{2} \frac{R_{3}}{R_{4}}+\mathrm{m}_{3}^{2} \frac{R_{4}}{R_{3}}\left[\left(M^{1}\right)^{2}+\left(M^{2}\right)^{2}\right]\right] \mathbf{M}_{i} \cdot \mathbf{M}_{i} \\
& -\frac{\pi^{2}}{2} \mathrm{~m}_{4}^{2} \frac{R_{3}}{R_{4}}\left(M^{1} M_{i}^{2}-M^{2} M_{i}^{1}+M^{3} M_{i}^{4}-M^{4} M_{i}^{3}\right)^{2} \\
& -\frac{\pi^{2}}{2} \mathrm{~m}_{3}^{2} \frac{R_{4}}{R_{3}}\left(M^{1} M_{i}^{2}-M^{2} M_{i}^{1}\right)^{2} \\
& +\frac{\pi^{2}}{2 R_{3} R_{4}} \mathrm{~m}_{4}^{2} \mathrm{~m}_{3}^{2}\left[\left(M^{1}\right)^{2}+\left(M^{2}\right)^{2}\right]\left[\left(M^{3}\right)^{2}+\left(M^{4}\right)^{2}\right] .
\end{aligned}
$$

We note again that the lowest energy states correspond to the lowest KK modes, being $\mathrm{m}_{4}= \pm 1$ and $\mathrm{m}_{3}= \pm 1$. The two signs are obviously not observable in the above Lagrangian (3.13). One may however ask whether what consequences a relative sign between the two momenta on the first compactified circle $\left(x^{4} \sim x^{4}+2 \pi R_{4}\right)$ may yield. Again it simply amounts to a sign in front of the last two terms (in the parenthesis) on the third line in eq. (3.13), which again can easily be compensated by renaming the two fields $\left\{M^{3}, M^{4}\right\} \rightarrow\left\{M^{4}, M^{3}\right\}$.

In the next section we will construct Euclidean solitons in the above Lagrangians.

\section{Euclidean solitons or instantons}

In this section we will consider Euclidean solitons in the supersymmetric Skyrme model and its derivatives coming from SS dimensional reduction.

\subsection{4d pure Skyrme instanton}

The first and simplest case is to consider a Euclidean soliton directly in the 4-dimensional theory (3.2), since the action is classically conformal in said number of dimensions. It is therefore an instanton-like soliton, first constructed in [31]. Note that the pure Skyrme model [31] and the bosonic sector of the NG restricted submanifold of the supersymmetric Skyrme model are identical. Hence the solution is directly applicable and here we will just make a swift review of the pure Skyrme-instanton. Let us start with the Lagrangian density (2.11) and use the Ansatz for the Skyrme field

$$
U=q \eta q^{-1}
$$

where $q$ is the identity map from $S^{3} \rightarrow \mathrm{SU}(2)$ as

$$
q=\hat{x}^{4} \mathbf{1}_{2}+i \tau^{a} \hat{x}^{a},
$$




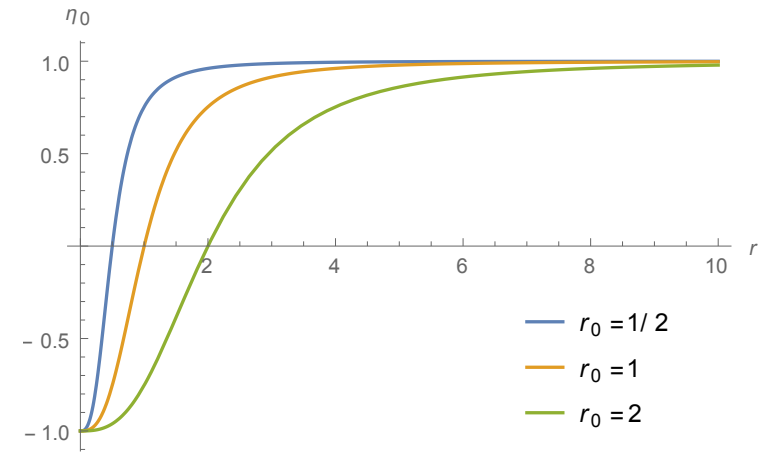

Figure 1. Skyrme-instanton solutions for various instanton sizes $r_{0}=e^{s_{0}}=1 / 2,1,2$.

where $\hat{x}^{\mu} \equiv x^{\mu} / r$ and the radius of the 3 -sphere is $r=\sqrt{x^{\mu} x^{\mu}}$ and finally

$$
\eta=\eta_{0}(r) \mathbf{1}_{2}+i \tau^{3} \eta_{3}(r)
$$

where $\eta$ is a curve in $\mathrm{SU}(2)$ obeying the constraint $\eta_{0}^{2}+\eta_{3}^{2}=1$. Thus the Euclidean action can be written as

$$
S_{E}=16 \pi^{2} \int d s\left[\frac{1}{2} \eta_{0}^{\prime 2}(s)+\left[1-\eta_{0}^{2}(s)\right]^{2}\right],
$$

where we have introduced $s=\log r$. The Skyrme-instanton solution is

$$
\eta_{0}(s)=\tanh \sqrt{2}\left(s-s_{0}\right)
$$

which solves both the second-order equation of motion derived from the Euclidean action (4.4) and the Bogomol'nyi equation

$$
\eta_{0}^{\prime}(s)=\sqrt{2}\left[1-\eta_{0}^{2}(s)\right] .
$$

The Euclidean action associated with the Skyrme-instanton solution (4.5) is

$$
S_{E}=16 \sqrt{2} \pi^{2} \int d s \eta_{0}^{\prime}(s)\left[1-\eta_{0}^{2}(s)\right]=16 \sqrt{2} \pi^{2} \int d \eta_{0}\left[1-\eta_{0}^{2}\right]=\frac{64 \sqrt{2} \pi^{2}}{3},
$$

where we have used the boundary conditions for the instanton solution: $\eta_{0}(-\infty)=-1$ and $\eta_{0}(\infty)=1$. The topological charge of the instanton is, however, $\mathbb{Z}_{2}$ [31]; to see this requires a suspension of the Hopf map to get to a nontrivial $\pi_{4}\left(S^{3}\right)$, see also e.g. [36].

In figure 1 we show 4d Skyrme-instanton solutions for various instanton sizes. Notice that $\eta_{0}\left(r_{0}\right)=0$, i.e. the curve in $\mathrm{SU}(2)$ passes zero exactly at the values of $r_{0}=e^{s_{0}}$ which we called the instanton size.

In the following, we look for instantons in three- and two-dimensional models.

\subsection{3d Skyrmion-instanton}

Skyrmions in the pure Skyrme theory (without a kinetic or potential term) are unstable against expanding themselves. Either a kinetic term or a potential term is needed to 
stabilize the Skyrmions with a finite size (and energy). Here, we have the kinetic term for the SS dimensionally reduced theory and we call Skyrmions in 3-dimensional Euclidean theory Skyrmion-instantons. After performing a single SS dimensional reduction to 3dimensional Euclidean space, we have the Lagrangian density (3.7). For simplicity, we will construct a soliton in the isotropically reduced theory, namely (3.8) which corresponds to the latter Lagrangian with the two momenta on the circle set equal $\mathrm{m}_{4,1}=\mathrm{m}_{4,2}$.

For convenience, we will rescale the lengths as $x^{a}=\frac{2 R_{4}}{\mathrm{~m}_{4}} \tilde{x}^{a}$, where $\tilde{x}^{a}$ are dimensionless coordinates. The Euclidean action thus reads

$$
\begin{aligned}
& S_{E}=\pi \mathrm{m}_{4} \int d^{3} \tilde{x} \mathcal{L}_{E} \\
& \mathcal{L}_{E}=\frac{1}{4}\left[\left(\mathbf{N}_{a} \cdot \mathbf{N}_{a}\right)^{2}-\left(\mathbf{N}_{a} \cdot \mathbf{N}_{b}\right)^{2}\right]+\frac{1}{2} \mathbf{N}_{a} \cdot \mathbf{N}_{a}-\frac{1}{2}\left[N^{1} N_{a}^{2}-N^{2} N_{a}^{1}+N^{3} N_{a}^{4}-N^{4} N_{a}^{3}\right]^{2} .
\end{aligned}
$$

The equations of motion derived from the above action read

$$
\begin{aligned}
N_{a a}^{\alpha} & +\left(\mathbf{N}_{b} \cdot \mathbf{N}_{b}\right) N_{a a}^{\alpha}+\left(\mathbf{N}_{a b} \cdot \mathbf{N}_{b}\right) N_{a}^{\alpha}-\left(\mathbf{N}_{a} \cdot \mathbf{N}_{b}\right) N_{a b}^{\alpha}-\left(\mathbf{N}_{b b} \cdot \mathbf{N}_{a}\right) N_{a}^{\alpha} \\
& +\left(N^{1} N_{a a}^{2}-N^{2} N_{a a}^{1}+N^{3} N_{a a}^{4}-N^{4} N_{a a}^{3}\right)\left(N^{2} \delta^{\alpha 1}-N^{1} \delta^{\alpha 2}+N^{4} \delta^{\alpha 3}-N^{3} \delta^{\alpha 4}\right) \\
& +2\left(N^{1} N_{a}^{2}-N^{2} N_{a}^{1}+N^{3} N_{a}^{4}-N^{4} N_{a}^{3}\right)\left(N_{a}^{2} \delta^{\alpha 1}-N_{a}^{1} \delta^{\alpha 2}+N_{a}^{4} \delta^{\alpha 3}-N_{a}^{3} \delta^{\alpha 4}\right)=0
\end{aligned}
$$

where $\alpha=1,2,3,4$. We will call the soliton a $3 \mathrm{~d}$ Skyrmion-instanton and it is very similar to a Skyrmion in the sense that it wraps a 3 -sphere in the target space and lives in 3dimensional (Euclidean) configuration space.

Since the last term in the action (4.8) breaks spherical symmetry we have not been able to reduce the equation of motion for a single 3d-Skyrmion-instanton to an ordinary differential equation. We therefore turn to numerical methods and solve the full partial differential equations with the finite difference method in conjunction with the relaxation method on an $81^{3}$ cubic lattice with a fourth-order stencil. We define the topological charge of the 3d-Skyrmion-instanton as

$$
B=-\frac{1}{2 \pi^{2}} \int d^{3} \tilde{x} \mathcal{B}, \quad \mathcal{B}=\frac{1}{6} \epsilon^{a b c} \epsilon^{\alpha \beta \gamma \delta} N_{a}^{\alpha} N_{b}^{\beta} N_{c}^{\gamma} N^{\delta} .
$$

The solution is shown as isosurfaces of the topological charge and Euclidean Lagrangian densities at their respective half-maximum values in figure 2. We have colored the figures using a normalized 3-vector $\mathbf{v} \equiv\left(N^{2}, N^{3}, N^{4}\right) / \sqrt{\left(N^{2}\right)^{2}+\left(N^{3}\right)^{2}+\left(N^{4}\right)^{2}}$ and mapping $v^{3}+$ $i v^{2}=e^{i \theta_{\text {color }}}$, where $\theta_{\text {color }}=0, \pi / 3,2 \pi / 3$ corresponds to red, green and blue, respectively. $v^{1}$ is then mapped to the lightness with $\left|v^{1}\right|=1$ being white and $v^{1}=0$ being black.

As we can see from the figure, the solution is a squashed sphere. In order to calculate the squashing of the solution, let us first define the size of the 3d-Skyrmion-instanton along the $x^{a}$ direction for a fixed $a$ as

$$
\left\langle\left(x^{a}\right)^{2}[\mathcal{X}]\right\rangle=\frac{\int d^{3} \tilde{x}\left(x^{a}\right)^{2} \mathcal{X}}{\int d^{3} \tilde{x} \mathcal{X}}
$$

where the index $a$ is not summed over and $\mathcal{X}$ is a density. The solution calculated in figure 2 is rotated such that there is an axial symmetry in the $\left(x^{1}, x^{3}\right)$-plane and thus we 


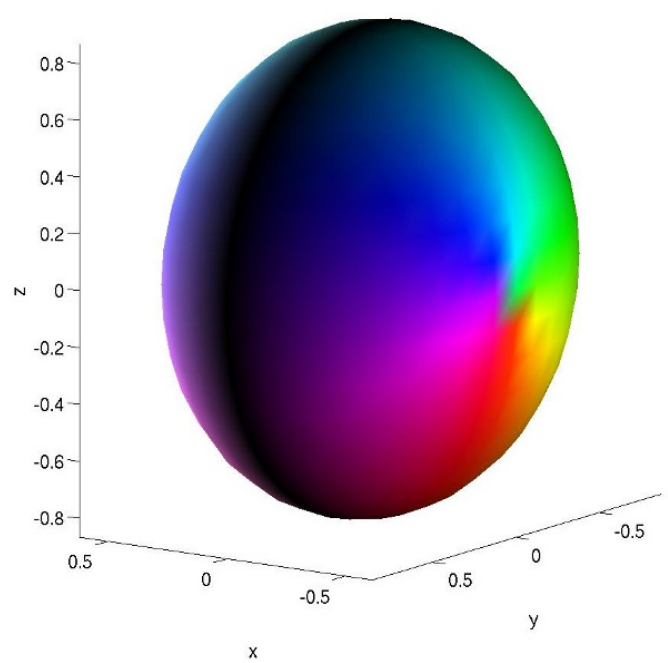

(a)

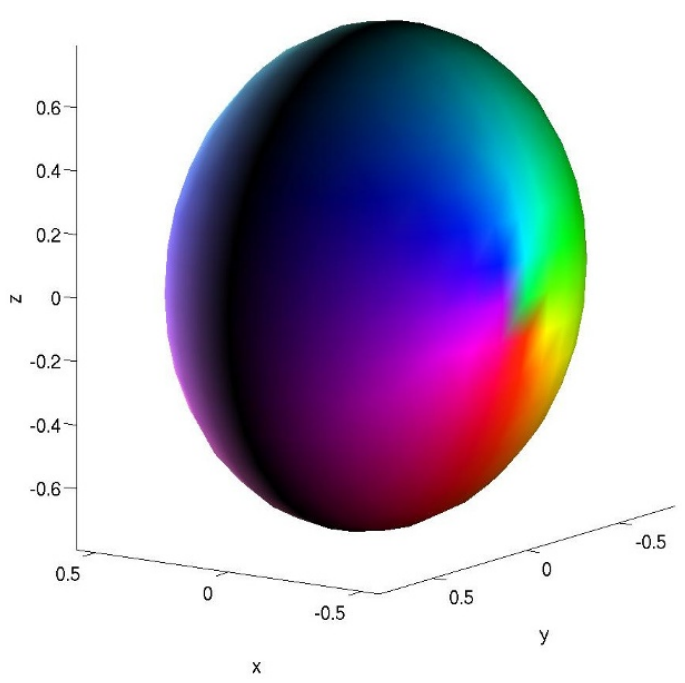

(b)

Figure 2. 3d-Skyrmion-instanton solution in the once SS reduced 3-Euclidean dimensional theory. (a) shows the isosurface of the topological charge density and (b) the isosurface of the Euclidean Lagrangian density; both at their respective half-maximum values. The solution is a squashed sphere with squashing parameter measured to be $\sigma[\mathcal{B}]=0.9499$ and $\sigma\left[\mathcal{L}_{E}\right]=0.9308$; the topological charge evaluated numerically as $B^{\text {numerical }}=0.9992$ and the Euclidean action is $S_{E}=\pi \mathrm{m}_{4} \times 55.91$. The colors are described in the text.

can define the squashing parameter as

$$
\sigma[\mathcal{X}] \equiv \sqrt{\frac{\left\langle\left(x^{2}\right)^{2}[\mathcal{X}]\right\rangle}{\left\langle\left(x^{1}\right)^{2}[\mathcal{X}]\right\rangle}}
$$

where we will use the topological charge density $\mathcal{X}=\mathcal{B}$ and the Euclidean Lagrangian density $\mathcal{X}=\mathcal{L}_{E}$, respectively; the numerical calculation gives $\sigma[\mathcal{B}]=0.9499$ and $\sigma\left[\mathcal{L}_{E}\right]=$ 0.9308 .

\section{$4.32 \mathrm{~d}$ vortex-instanton}

We will now consider the case of two consecutive SS dimensional reductions where the first one is isotropic and the second dimensional reduction is anisotropic in the way that only the first two fields depend nontrivially on the second circle coordinate. We thus have the case of the first reduction with equal momenta $\left(\mathrm{m}_{4,1}=\mathrm{m}_{4,2}=\mathrm{m}_{4}\right)$ and the second reduction with only one momentum $\left(\mathrm{m}_{3,1}=\mathrm{m}_{3}\right.$ and formally $\left.\mathrm{m}_{3,2}=0\right)$.

It will again prove convenient to rescale the lengths as $x^{i}=2 \sqrt{\frac{R_{3} R_{4}}{\mathrm{~m}_{3} \mathrm{~m}_{4}}} \tilde{x}^{i}$, where $\tilde{x}^{i}$ are dimensionless coordinates. The anisotropic dimensional reduction induces a potential which we need in order to construct a Euclidean vortex. We will employ the appropriate Ansatz for the vortex [37-39]

$$
\mathbf{M}=\{\cos f(r) \cos \alpha, \cos f(r) \sin \alpha, \sin f(r) \cos \theta, \sin f(r) \sin \theta\},
$$




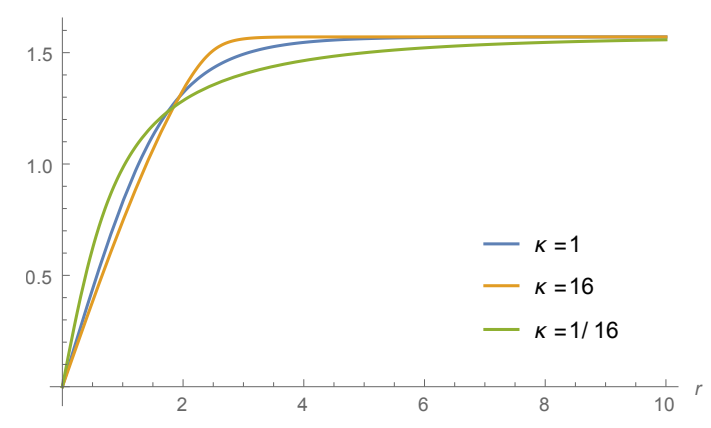

(a) $f$

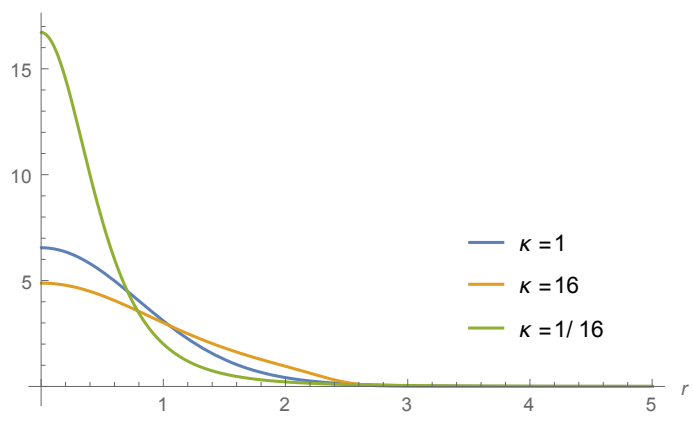

(b) $\mathcal{L}_{E}$

Figure 3. 2d vortex-instanton solutions for various values of $\kappa=1,16,1 / 16$. (a) shows the radial profile function $f$ and (b) the Euclidean Lagrangian density $\mathcal{L}_{E}$. The action of the solutions are indeed finite and are calculated numerically to be $S_{E}(1)=\pi^{3} \mathrm{~m}_{3} \mathrm{~m}_{4} \times 4.658, S_{E}(16)=\pi^{3} \mathrm{~m}_{3} \mathrm{~m}_{4} \times$ 5.241 and $S_{E}(1 / 16)=\pi^{3} \mathrm{~m}_{3} \mathrm{~m}_{4} \times 4.328$.

where $r e^{i \theta}=\tilde{x}^{1}+i \tilde{x}^{2}$ are the standard polar coordinates in two dimensions and $\alpha$ is a U(1) modulus. Finally, the 2-dimensional Euclidean action for the vortex system is found by plugging the above Ansatz into the Lagrangian (3.13) and it reads

$$
\begin{aligned}
& S_{E}=\pi^{3} \mathrm{~m}_{3} \mathrm{~m}_{4} \int d r r \mathcal{L}_{E}, \\
& \mathcal{L}_{E}=\frac{1}{r^{2}} \sin ^{2}(f) f_{r}^{2}+\left(\frac{1}{\kappa}+\kappa \cos ^{2} f\right)\left(f_{r}^{2}+\frac{1}{r^{2}} \sin ^{2} f\right)-\frac{1}{\kappa r^{2}} \sin ^{4} f+\cos ^{2} f \sin ^{2} f,
\end{aligned}
$$

where we have defined

$$
\kappa \equiv \frac{\mathrm{m}_{3} R_{4}}{\mathrm{~m}_{4} R_{3}}
$$

The winding number is defined as

$$
\mathcal{N}=\frac{1}{2 \pi} \int d r\left(M_{r}^{3} M_{\theta}^{4}-M_{r}^{4} M_{\theta}^{3}\right)=\frac{1}{2 \pi} \int d r \sin (2 f) f_{r}=\frac{1}{2 \pi} \int d f \sin 2 f=1,
$$

where we have used the boundary conditions $f(0)=0$ and $f(\infty)=\pi / 2$ in the last equality.

In figure 3 are shown the profile function $f$ and the Lagrangian density for various values of $\kappa=1 / 16,1,16$. Vortex solutions in this model are somewhat similar to those in the Skyrme model [37-39] with a similar potential term, albeit the kinetic term has a nontrivial field dependence as in $K$-theories, see e.g. [40-42].

Notice that although there are two potentially logarithmically divergent terms in the action, they come with coefficients $\kappa^{-1}$ and $-\kappa^{-1}$ and thus cancel, leaving the action integral convergent. Let us however examine the potential divergence in more detail. The asymptotic behavior of the profile function is

$$
f \sim \frac{\pi}{2}-A e^{-\sqrt{\kappa} r}
$$




\begin{tabular}{|l|l|l|}
\hline Dim. & Instantons & Relevant terms \\
\hline 4 & Pure Skyrme-instanton & Pure Skyrme term, no kinetic term \\
3 & Skyrmion-instanton & Kinetic and Skyrme terms \\
2 & Vortex-instanton & Kinetic, potential and Skyrme terms \\
\hline
\end{tabular}

Table 1. Instantons in various dimensions.

where $A \in \mathbb{R}_{>0}$ is an undetermined constant. Substituting into the Euclidean Lagrangian density (4.15), we get to leading order (i.e. approaching zero the slowest) at asymptotically large $r$ :

$$
\mathcal{L}_{E} \sim 2 A^{2} e^{-2 \sqrt{\kappa} r}\left(1+\frac{1}{\kappa r^{2}}+\frac{\kappa}{r^{2}}\right)+\mathcal{O}\left(e^{-4 \sqrt{\kappa} r}\right)
$$

which leaves the action integral convergent, as promised.

\section{Discussion and conclusion}

In this paper we have constructed solitons in the supersymmetric Skyrme model in three different codimensions from two through four. The results are summarized in table. 1. As mentioned, the supersymmetric Skyrme model lacks a kinetic term because the auxiliary field equation eliminates it, leaving only a potential term for the quasi-NG bosons behind. In this paper, we concentrated on the theory restricted to the NG submanifold only, for which the supersymmetric Skyrme term is exactly equal to the standard bosonic Skyrme term. First we reviewed Speight's instanton in the 4-dimensional Euclidean pure Skyrme model. Then we performed a Scherk-Schwarz dimensional reduction to 3 dimensions in which we constructed a 3-dimensional instanton that looks like a squashed sphere. Finally, we performed yet another anisotropic SS dimensional reduction to 2 dimensions, in which we constructed a Euclidean vortex-instanton with finite action. We would like to point out that this is - to the best of our knowledge - the first global vortex with finite tension.

As mentioned above, the existence of a stable soliton necessitates the existence of a pressure term. Two common terms are the kinetic term and the potential term. One possibility is to use the potential that is induced by the kinetic term; we mentioned that it does not provide a potential for the NG bosons, but only for the quasi-NG bosons: this is only if $G$-invariance (here it is $\mathrm{SU}(2) \times \mathrm{SU}(2))$ is kept. If we sacrifice the $G$-invariance, then we can make a $G$-symmetry breaking potential in the theory. Another possibility, yet to be explored, is to include a superpotential in the theory and solve the auxiliary field equation again [43].

A comment on supersymmetry of the models is in order. We have performed SS dimensional reduction on the pure Skyrme model which is just a truncation of the supersymmetric Skyrme model to the NG subspace. Although the solution of the auxiliary field has quite a non-linear form on the non-canonical branch [26], it is possible to write down the full untruncated Lagrangian and perform the SS dimensional reductions to three and two dimensions. We pointed out that the 4d Skyrme-instantons by Speight are not BPS in the supersymmetric model. This is obvious because of the fact that the topological charge 
is $\mathbb{Z}_{2}$ and hence not $\mathbb{Z}$. Consequently, two instantons annihilate each other and an antiinstanton is the same as the instanton itself. This is in contrast to BPS solitons for which two solitons have the mass exactly equal to twice the mass of a single soliton. Indeed, it is also possible to show that all the instantons discussed in this paper are non-BPS solitons. A brief discussion on the BPS properties of the instantons in three and two dimensions is found in appendix.

In this paper we have performed SS dimensional reductions, which can be obtained as the small compactification limit of the compactified circle with twisted boundary conditions (TBC). This reduction can give a relation between solitons in different dimensions.

For instance, this has been considered in Yang-Mills theory in $\mathbb{R}^{3} \times S^{1}$ [44-47], by which an instanton (caloron) is decomposed into a set of BPS monopoles.

Skyrme chains have been constructed in $\mathbb{R}^{2} \times S^{1}$ also with TBC, which for chains of 1Skyrmions are well-approximated by the holonomy of Yang-Mills calorons [48]. Instantons in the principal chiral model were constructed on $\mathbb{R}^{2} \times S^{1}$ with TBC in [49, 50]. A 3d instanton is first interpreted as a vortex ring with a $\mathrm{U}(1)$ modulus twisted once [37-39], and in the small compactification limit, it is decomposed into a vortex and an anti-vortex with the U(1) modulus twisted half (having half Skyrmion charges) [49, 50]. The vortexinstanton in this paper is somewhat similar to these two cases.

Lumps (sigma model instantons) in the $\mathbb{C} P^{n}$ model in $\mathbb{R}^{1} \times S^{1}$ with TBC were constructed in $[30,51,52]$. A lump can be interpreted as a domain wall ring with the $\mathrm{U}(1)$ modulus twisted once, and in small compactification limit it is decomposed into a kink and an anti-kink with U(1) modulus twisted half (having half lump charges) [30, 51, 52]. ${ }^{2}$

These relations should hold for our $4 \mathrm{~d}$ instanton and $3 \mathrm{~d}$ instanton. In four dimensions, a 3d Skyrmion-instanton is a string with $\mathrm{SU}(2)$ moduli. When we make a ring the $\mathrm{SU}(2)$ moduli should be twisted somehow to induce a $\pi_{4}$ charge. This was discussed in the context in Helium-3; a Shanker monopole string (characterized by $\pi_{3}[\mathrm{SO}(3)] \simeq \mathbb{Z}$ ) is twisted to make a ring producing an instanton (characterized by $\pi_{4}[\mathrm{SO}(3)] \simeq \mathbb{Z}_{2}$ ) [36]. Difficulties, however, are that $\mathrm{SU}(2)$ moduli cannot be uniquely twisted along $S^{1}$ and that the $\pi_{4}$ charge is $\mathbb{Z}_{2}$ so twisting twice should be equivalent to untwisting.

A story, similar to the one in this paper, plays out in the supersymmetric baby Skyrme model [23, 24, 58-60], where the kinetic term also vanishes when a nontrivial solution of the auxiliary field equation is used [23]. Analogously to this paper, Scherk-Schwarz dimensional reduction can be carried out also in that case, yielding a kinetic term and possibly a domain wall solution $[51,52,57]$ if we perform it twice.

\section{Acknowledgments}

S. B. G. thanks the Recruitment Program of High-end Foreign Experts for support. The work of S. B. G. was supported by the National Natural Science Foundation of China (Grant No. 11675223). The work of M. N. is supported in part by a Grant-in-Aid for

\footnotetext{
${ }^{2}$ This was used to construct bions, a pair of a kink-instanton and an anti-kink-instanton with zero total instanton charge (exact bion solutions are available for the $\mathbb{C} P^{2}$ model $[53,54]$ ). The application of bions to resurgence has been extensively studied [55-57].
} 
Scientific Research on Innovative Areas "Topological Materials Science" (KAKENHI Grant No. 15H05855) and "Nuclear Matter in Neutron Stars Investigated by Experiments and Astronomical Observations" (KAKENHI Grant No. 15H00841) from the the Ministry of Education, Culture, Sports, Science (MEXT) of Japan. The work of M. N. is also supported in part by the Japan Society for the Promotion of Science (JSPS) Grant-in-Aid for Scientific Research (KAKENHI Grant No. 25400268) and by the MEXT-Supported Program for the Strategic Research Foundation at Private Universities "Topological Science" (Grant No. S1511006). The work of S. S. is supported in part by Kitasato University Research Grant for Young Researchers.

\section{A BPS property of instanton solutions}

We remark that solitons in supersymmetric field theories are potentially BPS states which preserve fractions of supersymmetry. BPS states in 4-dimensional supersymmetric higherderivative theories have been classified in [25]. The BPS states satisfy the condition that the supersymmetry variation of $\psi_{M}$, the fermionic partner of $M$, vanishes for some $\xi$ and $\bar{\xi}$ :

$$
\delta\left(\psi_{M}\right)_{\alpha}=\sqrt{2} i\left(\sigma_{\mathrm{E}}^{\mu}\right)_{\alpha \dot{\alpha}} \bar{\xi}^{\dot{\alpha}} \partial_{\mu} M+\sqrt{2} \xi_{\alpha} F_{M}=0,
$$

where $\alpha=1,2$ and $\sigma_{\mathrm{E}}^{\mu}=\left(i \vec{\tau}, \mathbf{1}_{2}\right), \bar{\sigma}_{\mathrm{E}}^{\mu}=\left(-i \vec{\tau}, \mathbf{1}_{2}\right)$ which satisfy $\left\{\sigma_{\mathrm{E}}^{\mu}, \bar{\sigma}_{\mathrm{E}}^{\nu}\right\}=2 \delta^{\mu \nu} \mathbf{1}_{2}$ are the sigma matrices in Euclidean space. More explicitly, the variation is found to be

$$
\begin{aligned}
& \delta \psi_{M}=\sqrt{2}\left(\begin{array}{c}
\left(\partial_{3}-i \partial_{4}\right) M \bar{\xi}^{\dot{1}}+\left(\partial_{1}-i \partial_{2}\right) M \bar{\xi}^{\dot{2}}-i \xi_{1} F_{M} \\
\left(\partial_{3}+i \partial_{4}\right) M \bar{\xi}^{2}+\left(\partial_{1}+i \partial_{2}\right) M \bar{\xi}^{\mathrm{i}}-i \xi_{2} F_{M}
\end{array}\right) \\
& \delta \bar{\psi}_{M}=-\sqrt{2} i\left(\begin{array}{c}
\left(\partial_{3}+i \partial_{4}\right) \bar{M} \xi_{1}+\left(\partial_{1}-i \partial_{2}\right) \bar{M} \xi_{2}+i \bar{\xi}^{\dot{1}} \bar{F}_{M} \\
-\left(\partial_{3}-i \partial_{4}\right) \bar{M} \xi_{2}+\left(\partial_{1}+i \partial_{2}\right) \bar{M} \xi_{1}+i \bar{\xi}^{2} \bar{F}_{M}
\end{array}\right) .
\end{aligned}
$$

We stress that the supersymmetry transformation parameters $\xi$ and $\bar{\xi}$ are independent of each other in Euclidean spaces.

We are interested in codimension-four instanton-like configurations. For the noncanonical branch, we have $F_{M}, \bar{F}_{M} \neq 0$. Let us consider the following 1/4-BPS condition $\bar{\xi}^{\dot{1}} \neq 0, \bar{\xi}^{\dot{2}}=\xi_{1}=\xi_{2}=0$, then we have

$$
\left(\partial_{3}-i \partial_{4}\right) M=\left(\partial_{1}+i \partial_{2}\right) M=0, \quad \bar{F}_{M}=0 .
$$

The last condition together with the equation of motion of the auxliary field (2.8) with $f_{\pi}=0$ implies the following condition:

$$
0=\operatorname{Tr} F_{M} F_{M}^{\dagger}=\operatorname{Tr} \partial_{\mu} M \partial_{\mu} M^{\dagger} .
$$

The only solution to these conditions is $M=$ const. The other choices of nonzero component of $\xi, \bar{\xi}$ result in the same condition. The other possible 1/4-BPS combinations of $\xi, \bar{\xi}$, for example $\bar{\xi}^{\dot{2}}-i \xi_{1}=\bar{\xi}^{\mathrm{i}}=\xi_{2}=0$, lead to the condition

$$
\left(\partial_{1}-i \partial_{2}\right) M=F_{M}, \quad\left(\partial_{3}+i \partial_{4}\right) M=0 .
$$


This implies that $M$ is a holomorphic function of $z=x^{3}+i x^{4}$ and its dependence in the $x^{1}, x^{2}$ plane is determined by the source term $F_{M}$ on the right-hand side in the first equation. This is just a vortex-lump type configuration discussed in [25]. The combinations like $\bar{\xi}^{\dot{2}}-i \xi_{1}=\bar{\xi}^{i}-i \xi_{2}=0$ give lump-lump type configurations. This is true even if we restrict the model to the NG subspace. Therefore, there are no non-trivial $1 / 4$ BPS instantons in the pure Skyrme model in four dimensions.

For the $1 / 2$-BPS condition, if we keep two of the four parameters, it inevitably leads to the condition $F_{M}=0$ which results in a vacuum condition of $M$. If we combine, for example, $\bar{\xi}^{\dot{2}}=i \xi_{1}, \bar{\xi}^{\dot{1}}=i \xi_{2}$, this leads to the condition that $M$ is independent of $x^{3}, x^{4}$. Therefore codimension-four solitons are inconsistent with the $1 / 2$ BPS condition. We note that this is the story for codimension-four solitons. We can, however, find $1 / 4$ and $1 / 2$ BPS configurations of codimension two on the non-canonical branch [25].

The conclusion is that the pure Skyrme-instantons constructed by Speight is not BPS in the supersymmetric Skyrme model. In hindsight, it is obvious that they cannot be BPS since they carry $\mathbb{Z}_{2}$ charge. Although it is not a BPS solution in the sense that it does not preserve fractions of supersymmetry, the instantons by Speight in four dimensions do satisfy the equation of motion and saturate an energy bound on the NG subspace.

The same analysis can be applied even to the lower-dimensional models. In lower dimensions, the supersymmetry variation of fermions is given by eq. (A.2) in which the derivatives in the compactified directions $x^{3}, x^{4}$ are replaced by Killing vectors $G$ associated with isometries in the target space. Namely, the derivative with respect to the compactified direction induces a motion along the Killing vectors, e.g. $\partial_{3} M=m G(M)$ where $m$ is a mass parameter. These Killing vectors generate central charges in the supersymmetry algebra. Using this fact, it is obvious that the above discussion in four dimensions holds true in lower dimensions. We find that only possible BPS instantons are two-dimensional 1/2 BPS lumps in the massless theory, which are characterized by a holomorphic dependence on $x^{1}, x^{2}$. One finds that the Skyrmion-instanton and the vortex-instanton discussed in the main body of this paper do not belong to this class and they are thus non-BPS solitons.

Open Access. This article is distributed under the terms of the Creative Commons Attribution License (CC-BY 4.0), which permits any use, distribution and reproduction in any medium, provided the original author(s) and source are credited.

\section{References}

[1] T.H.R. Skyrme, A unified field theory of mesons and baryons, Nucl. Phys. 31 (1962) 556.

[2] T.H.R. Skyrme, A nonlinear field theory, Proc. Roy. Soc. Lond. A 260 (1961) 127.

[3] E. Witten, Global aspects of current algebra, Nucl. Phys. B 223 (1983) 422 [INSPIRE].

[4] E. Witten, Current algebra, baryons and quark confinement, Nucl. Phys. B 223 (1983) 433 [INSPIRE].

[5] R.A. Battye, N.S. Manton, P.M. Sutcliffe and S.W. Wood, Light nuclei of even mass number in the Skyrme model, Phys. Rev. C 80 (2009) 034323 [arXiv:0905.0099] [INSPIRE]. 
[6] P.H.C. Lau and N.S. Manton, States of Carbon-12 in the Skyrme model, Phys. Rev. Lett. 113 (2014) 232503 [arXiv: 1408.6680] [INSPIRE].

[7] C.J. Halcrow and N.S. Manton, A Skyrme model approach to the spin-orbit force, JHEP 01 (2015) 016 [arXiv: 1410.0880] [inSPIRE].

[8] M. Karliner, C. King and N.S. Manton, Electron scattering intensities and Patterson functions of skyrmions, J. Phys. G 43 (2016) 055104 [arXiv: 1510.00280] [INSPIRE].

[9] M. Haberichter, P.H.C. Lau and N.S. Manton, Electromagnetic transition strengths for light nuclei in the Skyrme model, Phys. Rev. C 93 (2016) 034304 [arXiv:1510.08811] [INSPIRE].

[10] P. Sutcliffe, Skyrmions, instantons and holography, JHEP 08 (2010) 019 [arXiv:1003.0023] [INSPIRE].

[11] P. Sutcliffe, Skyrmions in a truncated BPS theory, JHEP 04 (2011) 045 [arXiv:1101.2402] [INSPIRE].

[12] L.D. Faddeev, Some comments on the many dimensional solitons, Lett. Math. Phys. 1 (1976) 289 [INSPIRE].

[13] E.B. Bogomolny, Stability of classical solutions, Sov. J. Nucl. Phys. 24 (1976) 449 [Yad. Fiz. 24 (1976) 861] [INSPIRE].

[14] N.S. Manton and P.J. Ruback, Skyrmions in flat space and curved space, Phys. Lett. B 181 (1986) 137 [INSPIRE].

[15] C. Adam, J. Sanchez-Guillen and A. Wereszczynski, A Skyrme-type proposal for baryonic matter, Phys. Lett. B 691 (2010) 105 [arXiv:1001.4544] [InSPIRE].

[16] C. Adam, J. Sanchez-Guillen and A. Wereszczynski, A BPS Skyrme model and baryons at large- $N_{c}$, Phys. Rev. D 82 (2010) 085015 [arXiv:1007.1567] [inSPIRE].

[17] J.M. Queiruga, Skyrme-like models and supersymmetry in $3+1$ dimensions, Phys. Rev. D 92 (2015) 105012 [arXiv: 1508.06692] [INSPIRE].

[18] B. Zumino, Supersymmetry and Kähler manifolds, Phys. Lett. B 87 (1979) 203 [InSPIRE].

[19] E.A. Bergshoeff, R.I. Nepomechie and H.J. Schnitzer, Supersymmetric Skyrmions in four-dimensions, Nucl. Phys. B 249 (1985) 93 [InSPIRE].

[20] T. Fujimori, M. Nitta and Y. Yamada, Ghostbusters in higher derivative supersymmetric theories: who is afraid of propagating auxiliary fields?, JHEP 09 (2016) 106 [arXiv: 1608.01843] [INSPIRE].

[21] L. Freyhult, The supersymmetric extension of the Faddeev model, Nucl. Phys. B 681 (2004) 65 [hep-th/0310261] [INSPIRE].

[22] J. Khoury, J.-L. Lehners and B. Ovrut, Supersymmetric $P(X, \phi)$ and the ghost condensate, Phys. Rev. D 83 (2011) 125031 [arXiv: 1012.3748] [INSPIRE].

[23] C. Adam, J.M. Queiruga, J. Sanchez-Guillen and A. Wereszczynski, Extended supersymmetry and BPS solutions in baby Skyrme models, JHEP 05 (2013) 108 [arXiv:1304.0774] [INSPIRE].

[24] M. Nitta and S. Sasaki, BPS states in supersymmetric chiral models with higher derivative terms, Phys. Rev. D 90 (2014) 105001 [arXiv:1406.7647] [INSPIRE].

[25] M. Nitta and S. Sasaki, Classifying BPS states in supersymmetric gauge theories coupled to higher derivative chiral models, Phys. Rev. D 91 (2015) 125025 [arXiv:1504.08123] [INSPIRE]. 
[26] S.B. Gudnason, M. Nitta and S. Sasaki, A supersymmetric Skyrme model, JHEP 02 (2016) 074 [arXiv: 1512.07557] [INSPIRE].

[27] M. Nitta and S. Sasaki, Higher derivative corrections to manifestly supersymmetric nonlinear realizations, Phys. Rev. D 90 (2014) 105002 [arXiv:1408.4210] [InSPIRE].

[28] J. Scherk and J.H. Schwarz, Spontaneous breaking of supersymmetry through dimensional reduction, Phys. Lett. B 82 (1979) 60 [InSPIRE].

[29] J. Scherk and J.H. Schwarz, How to get masses from extra dimensions, Nucl. Phys. B 153 (1979) 61 [INSPIRE].

[30] M. Eto, Y. Isozumi, M. Nitta, K. Ohashi and N. Sakai, Solitons in the Higgs phase: the moduli matrix approach, J. Phys. A 39 (2006) R315 [hep-th/0602170] [INSPIRE].

[31] J.M. Speight, A pure Skyrme instanton, Phys. Lett. B 659 (2008) 429 [hep-th/0703198] [INSPIRE].

[32] M. Bando, T. Kuramoto, T. Maskawa and S. Uehara, Structure of nonlinear realization in supersymmetric theories, Phys. Lett. B 138 (1984) 94 [INSPIRE].

[33] M. Bando, T. Kuramoto, T. Maskawa and S. Uehara, Nonlinear realization in supersymmetric theories, Prog. Theor. Phys. 72 (1984) 313 [INSPIRE].

[34] M. Bando, T. Kuramoto, T. Maskawa and S. Uehara, Nonlinear realization in supersymmetric theories. 2, Prog. Theor. Phys. 72 (1984) 1207 [INSPIRE].

[35] A.C.W. Kotcheff and G.M. Shore, Kähler $\sigma$ models from supersymmetric gauge theories, Int. J. Mod. Phys. A 4 (1989) 4391 [inSPIRE].

[36] M. Nakahara, Toy skyrmions in superfluid He-3 A, Prog. Theor. Phys. 77 (1987) 1011 [INSPIRE].

[37] S.B. Gudnason and M. Nitta, Effective field theories on solitons of generic shapes, Phys. Lett. B 747 (2015) 173 [arXiv: 1407.2822] [INSPIRE].

[38] S.B. Gudnason and M. Nitta, Incarnations of Skyrmions, Phys. Rev. D 90 (2014) 085007 [arXiv:1407.7210] [INSPIRE].

[39] S.B. Gudnason and M. Nitta, Skyrmions confined as beads on a vortex ring, Phys. Rev. D 94 (2016) 025008 [arXiv: 1606.00336] [INSPIRE].

[40] E. Babichev, Gauge k-vortices, Phys. Rev. D 77 (2008) 065021 [arXiv:0711.0376] [INSPIRE].

[41] C. Adam, P. Klimas, J. Sanchez-Guillen and A. Wereszczynski, Compact gauge $K$ vortices, J. Phys. A 42 (2009) 135401 [arXiv:0811.4503] [InSPIRE].

[42] D. Bazeia, E. da Hora, C. dos Santos and R. Menezes, BPS solutions to a generalized Maxwell-Higgs model, Eur. Phys. J. C 71 (2011) 1833 [arXiv:1201.2974] [INSPIRE].

[43] S. Sasaki, M. Yamaguchi and D. Yokoyama, Supersymmetric DBI inflation, Phys. Lett. B 718 (2012) 1 [arXiv:1205.1353] [INSPIRE].

[44] K.-M. Lee and P. Yi, Monopoles and instantons on partially compactified D-branes, Phys. Rev. D 56 (1997) 3711 [hep-th/9702107] [INSPIRE].

[45] T.C. Kraan and P. van Baal, Periodic instantons with nontrivial holonomy, Nucl. Phys. B 533 (1998) 627 [hep-th/9805168] [INSPIRE].

[46] T.C. Kraan and P. van Baal, Monopole constituents inside $\mathrm{SU}(N)$ calorons, Phys. Lett. B 435 (1998) 389 [hep-th/9806034] [INSPIRE]. 
[47] E.J. Weinberg and P. Yi, Magnetic monopole dynamics, supersymmetry and duality, Phys. Rept. 438 (2007) 65 [hep-th/0609055] [INSPIRE].

[48] D. Harland and R.S. Ward, Chains of Skyrmions, JHEP 12 (2008) 093 [arXiv:0807.3870] [INSPIRE].

[49] M. Nitta, Fractional instantons and bions in the $O(N)$ model with twisted boundary conditions, JHEP 03 (2015) 108 [arXiv: 1412.7681] [INSPIRE].

[50] M. Nitta, Fractional instantons and bions in the principal chiral model on $\mathbb{R}^{2} \times S^{1}$ with twisted boundary conditions, JHEP 08 (2015) 063 [arXiv:1503.06336] [INSPIRE].

[51] M. Eto, Y. Isozumi, M. Nitta, K. Ohashi and N. Sakai, Instantons in the Higgs phase, Phys. Rev. D 72 (2005) 025011 [hep-th/0412048] [INSPIRE].

[52] M. Eto et al., Non-abelian vortices on cylinder: duality between vortices and walls, Phys. Rev. D 73 (2006) 085008 [hep-th/0601181] [INSPIRE].

[53] R. Dabrowski and G.V. Dunne, Fractionalized non-self-dual solutions in the $C P^{N-1}$ model, Phys. Rev. D 88 (2013) 025020 [arXiv:1306.0921] [INSPIRE].

[54] T. Misumi, M. Nitta and N. Sakai, Non-BPS exact solutions and their relation to bions in $\mathbb{C} P^{N-1}$ models, JHEP 05 (2016) 057 [arXiv: 1604.00839] [InSPIRE].

[55] G.V. Dunne and M. Ünsal, Resurgence and trans-series in quantum field theory: the $C P^{N-1}$ model, JHEP 11 (2012) 170 [arXiv:1210.2423] [INSPIRE].

[56] G.V. Dunne and M. Ünsal, Continuity and resurgence: towards a continuum definition of the $\mathbb{C P}^{N-1}$ model, Phys. Rev. D 87 (2013) 025015 [arXiv:1210.3646] [INSPIRE].

[57] T. Misumi, M. Nitta and N. Sakai, Neutral bions in the $\mathbb{C} P^{N-1}$ model, JHEP 06 (2014) 164 [arXiv: 1404.7225] [INSPIRE].

[58] C. Adam, J.M. Queiruga, J. Sanchez-Guillen and A. Wereszczynski, $N=1$ supersymmetric extension of the baby Skyrme model, Phys. Rev. D 84 (2011) 025008 [arXiv:1105.1168] [INSPIRE].

[59] S. Bolognesi and W. Zakrzewski, Baby Skyrme model, near-BPS approximations and supersymmetric extensions, Phys. Rev. D 91 (2015) 045034 [arXiv: 1407.3140] [INSPIRE].

[60] J.M. Queiruga, Baby Skyrme model and fermionic zero modes, Phys. Rev. D 94 (2016) 065022 [arXiv: 1606.02869] [INSPIRE]. 